\title{
COMPLETELY CONVEX FUNCTIONS AND LIDSTONE SERIES
}

\author{
BY \\ D. V. WIDDER
}

Introduction. R. P. Boas suggested to the author the possibility of using Lidstone series to discuss the analytic character of functions whose even derivatives are positive on an interval. The author has described in an earlier note $\left.{ }^{1}\right)$ how this suggestion led him to prove that a function for which

$$
(-1)^{n} f^{(2 n)}(x) \geqq 0 \quad(n=0,1,2, \cdots)
$$

on an interval is necessarily entire. In that note it was stated that we would show later the relation of this result to the problem of the representation of functions by Lidstone series. It is the aim of the present paper to study this relation.

A Lidstone series is a generalization of Taylor's series. It approximates to a given function in the neighborhood of two points instead of one. Such series have been studied by G. J. Lidstone $\left({ }^{2}\right)$, H. Poritsky $\left({ }^{3}\right)$, J. M. Whittaker $\left({ }^{4}\right)$, I. J. Schoenberg $\left({ }^{5}\right)$ and others. More precisely the series has the form

(2) $f(x)=f(1) \Lambda_{0}(x)+f(0) \Lambda_{0}(1-x)+f^{\prime \prime}(1) \Lambda_{1}(x)+f^{\prime \prime}(0) \Lambda_{1}(1-x)+\cdots$,

where $\Lambda_{n}(x)$ is a polynomial of degree $2 n+1$ defined by the relations

$$
\begin{aligned}
\Lambda_{0}(x) & =x, \\
\Lambda_{n}^{\prime \prime}(x) & =\Lambda_{n-1}(x), \\
\Lambda_{n}(0) & =\Lambda_{n}(1)=0 \quad(n=1,2, \cdots) .
\end{aligned}
$$

Thus it is clear that the sum of an even number of terms of the series (2) is a polynomial which coincides with $f(x)$ at $x=0$ and at $x=1$. Moreover, each even derivative of the polynomial which does not vanish identically coincides with the corresponding derivative of $f(x)$ at those points.

Presented to the Society, January 1, 1941, under the title Necessary and sufficient conditions for the representation of Lidstone series; received by the editors February 21, 1941.

(1) D. V. Widder, Functions whose even derivatives have a prescribed sign, Proceedings of the National Academy of Sciences, vol. 26 (1940), pp. 657-659.

(2) G. J. Lidstone, Notes on the extension of Aitken's theorem (for polynomial interpolation) to the Everett types, Proceedings of the Edinburgh Mathematical Society, (2), vol. 2 (1929), pp. 16-19.

(3) H. Poritsky, On certain polynomial and other approximations to analytic functions, these Transactions, vol. 34 (1932), pp. 274-331.

(4) J. M. Whittaker, On Lidstone's series and two-point expansions of analytic functions. Proceedings of the London Mathematical Society, vol. 36 (1934), pp. 451-469.

(5) I. J. Schoenberg, On certain two-point expansions of integral functions of exponential type, Bulletin of the American Mathematical Society, vol. 42 (1936), pp. 284-288. 
Boas has suggested, following the analogy with the completely monotonic functions of S. Bernstein, that we call functions satisfying (1) completely con$v e x$. We adopt this terminology and show that any function which is completely convex in an interval of length greater than $\pi$ has an expansion (2) with every term positive. Since the sum of a Lidstone series must be entire we have here a new proof of the result mentioned above.

This sufficient condition for expansion in Lidstone series is not necessary, as simple examples show. In earlier papers, necessary conditions and sufficient conditions have been obtained, but none that are both necessary and sufficient. In the present paper such conditions are obtained by introducing the class of minimal completely convex functions, a slight variation of the class described above. It is proved, in fact, that a real function can be expanded in an absolutely convergent Lidstone series if and only if it is the difference of two minimal completely convex functions.

1. The Green's function of a certain differential system. Define a function $G(x, t)$ as follows

$$
\begin{aligned}
G(x, t) & =(x-1) t & & (0 \leqq t<x \leqq 1), \\
& =(t-1) x & & (0 \leqq x \leqq t \leqq 1) .
\end{aligned}
$$

If $\phi(x)$ is any function continuous in the interval $0 \leqq x \leqq 1$, then it is easily verified that the unique solution of the differential system,

$$
\begin{aligned}
f^{\prime \prime}(x) & =\phi(x), \\
f(0) & =f(1)=0,
\end{aligned}
$$

is

$$
f(x)=\int_{0}^{1} G(x, t) \phi(t) d t .
$$

Consider next the successive iterates of $G(x, t)$, defined by the equations

$$
\begin{aligned}
G_{1}(x, t) & =G(x, t), \\
G_{n}(x, t) & =\int_{0}^{1} G(x, y) G_{n-1}(y, t) d y \quad(n=2,3, \cdots) .
\end{aligned}
$$

From the property of $G(x, t)$ described above it is now clear that the function,

$$
f(x)=\int_{0}^{1} G_{n}(x, t) \phi(t) d t,
$$

is the unique solution of the differential system

$$
\begin{aligned}
& f^{(2 n)}(x)=\phi(x), \\
& f^{(2 k)}(0)=f^{(2 k)}(1)=0 \quad(k=0,1, \cdots, n-1) .
\end{aligned}
$$


In fact $G_{n}(x, t)$ is the familiar Green's function of the system. Observe that $(-1)^{n} G_{n}(x, t)$ is non-negative in the unit square $0 \leqq x \leqq 1,0 \leqq t \leqq 1$.

Finally we see that the unique solution of the system

$$
f^{(2 n)}(x)=\phi(x), f^{(2 k)}(0)=a_{2 k}, \quad f^{(2 k)}(1)=b_{2 k}(k=0,1, \cdots, n-1),
$$
is

$$
\begin{aligned}
f(x)=a_{0}(1-x)+b_{0} x & +\sum_{k=1}^{n-1} a_{2 k} \int_{0}^{1} G_{k}(x, t)(1-t) d t \\
& +b_{2 k} \int_{0}^{1} G_{k}(x, t) t d t+R_{n}(x)
\end{aligned}
$$

where

$$
R_{n}(x)=\int_{0}^{1} G_{n}(x, t) \phi(t) d t
$$

If in this equation we replace $a_{2 k}, b_{2 k}$ and $\phi(x)$ by their values in terms of $f(x)$ as given by the differential system (1.3) we obtain an identity which holds for all functions $f(x)$ possessing a sufficient number of derivatives. In fact this identity can be obtained without any appeal to the theory of differential equations by integrating the last integral by parts. We state the result as a theorem.

THEOREM 1.1. If $f(x)$ is a function of class $C^{2 n}$ in the interval $0 \leqq x \leqq 1$, then

$$
\begin{aligned}
f(x)= & f(0)(1-x)+f(1) x+\sum_{k=1}^{n-1} f^{(2 k)}(0) \int_{0}^{1} G_{k}(x, t)(1-t) d t \\
& +f^{(2 k)}(1) \int_{0}^{1} G_{k}(x, t) t d t+R_{n}(x),
\end{aligned}
$$

where

$$
R_{n}(x)=\int_{0}^{1} G_{n}(x, t) f^{(2 n)}(t) d t
$$

2. Certain Fourier expansions. Starting with the familiar Fourier series

$$
1=\frac{4}{\pi} \sum_{k=0}^{\infty} \frac{\sin (2 k+1) \pi t}{(2 k+1)} \quad(0<t<1),
$$

we multiply the series by $G_{1}(x, t)$ and integrate with respect to $t$ from zero to unity. By (1.1) and (1.2) we have

$$
\int_{0}^{1} G_{1}(x, t) \sin (2 k+1) \pi t d t=-\frac{\sin (2 k+1) \pi x}{(2 k+1)^{2} \pi^{2}},
$$


so that we obtain at once the Fourier expansion of $\int_{0}^{1} G_{1}(x, t) d t$. Repeating the process $n$ times we obtain

THEOREM 2.1. For $0 \leqq x \leqq 1$ and $n=1,2, \cdots$ the following expansion holds:

$$
\int_{0}^{1} G_{n}(x, t) d t=(-1)^{n} \frac{4}{\pi^{2 n+1}} \sum_{k=0}^{\infty} \frac{\sin (2 k+1) \pi x}{(2 k+1)^{2 n+1}} .
$$

A result of a similar nature is obtained from the familiar series

$$
t=\frac{2}{\pi} \sum_{k=1}^{\infty} \frac{(-1)^{k+1}}{k} \sin k \pi t
$$

We record the result in

TheOREM 2.2. For $0 \leqq x \leqq 1$ and $n=1,2, \cdots$ the following expansion holds:

$$
\int_{0}^{1} G_{n}(x, t) t d t=(-1)^{n} \frac{2}{\pi^{2 n+1}} \sum_{k=1}^{\infty} \frac{(-1)^{k+1}}{k^{2 n+1}} \sin k \pi x .
$$

3. Lidstone polynomials. The function (2.2) is clearly a polynomial of degree $2 n+1$ since its derivative of order $2 n$ is the function $x$. We shall refer to it as the Lidstone polynomial of order $n$ and, following the original notation of Lidstone, set

$$
\begin{aligned}
& \Lambda_{0}(x)=x, \\
& \Lambda_{n}(x)=\int_{0}^{1} G_{n}(x, t) t d t \quad(n=1,2, \cdots) .
\end{aligned}
$$

By subtracting equation (2.2) from equation (2.1) we have

$$
\int_{0}^{1} G_{n}(x, t)(1-t) d t=(-1)^{n} \frac{2}{\pi^{2 n+1}} \sum_{k=1}^{\infty} \frac{\sin k \pi x}{k^{2 n+1}} .
$$

But if we replace $x$ by $(1-x)$ in this series we obtain the series (2.2), whose sum is $\Lambda_{n}(x)$. That is, the equation

$$
\int_{0}^{1} G_{n}(x, t)(1-t) d t=\Lambda_{n}(1-x)
$$

holds at least for $0 \leqq x \leqq 1$. But the result is true for all $x$ since both sides of the equation are polynomials. Equations (1.4) and (2.2) now become

(3.1) $f(x)=\sum_{k=0}^{n-1}\left[f^{(2 k)}(0) \Lambda_{k}(x)+f^{(2 k)}(1) \Lambda_{k}(1-x)\right]+\int_{0}^{1} G_{n}(x, t) f^{(2 n)}(t) d t$,

(3.2) $\Lambda_{n}(x)=(-1)^{n} \frac{2}{\pi^{2 n+1}} \sum_{k=1}^{\infty} \frac{(-1)^{k+1}}{k^{2 n+1}} \sin k \pi x$

$(0 \leqq x \leqq 1)$. 
4. Asymptotic behavior of $\Lambda_{n}(x)$ for large $n$. The first term of the Fourier expansion (3.2) serves as a close approximation to $\Lambda_{n}(x)$ if $n$ is large. More explicitly we have

THEOREM 4.1. There exists a constant $M$ such that

$$
\left|(-1)^{n} \Lambda_{n}(x)-\frac{2}{\pi^{2 n+1}} \sin \pi x\right|<\frac{M}{(2 \pi)^{2 n+1}} \quad(0 \leqq x \leqq 1 ; n=0,1, \cdots) .
$$

For, it is clear from equation (3.2) that for $0 \leqq x \leqq 1$,

$$
\left|(-1)^{n} \Lambda_{n}(x)-\frac{2}{\pi^{2 n+1}} \sin \pi x\right| \leqq \frac{2}{(2 \pi)^{2 n+1}}\left[1+\left(\frac{2}{3}\right)^{2 n+1}+\left(\frac{2}{4}\right)^{2 n+1}+\cdots\right] .
$$

The result is now obvious since the Dirichlet series in brackets tends to unity when $n$ becomes infinite.

THEOREM 4.2. There exists a constant $M$ such that

$$
0 \leqq(-1)^{n} \Lambda_{n}(x) \leqq \frac{M}{\pi^{2 n}} \quad(0 \leqq x \leqq 1 ; n=1,2, \cdots) .
$$

This follows at once from (4.1).

Theorem 4.3. For any fixed $x_{0}$ between zero and unity there is a constant $M$ such that

$$
(-1)^{n} \Lambda_{n}\left(x_{0}\right) \geqq \frac{M}{\pi^{2 n}} \quad(n=1,2, \cdots) .
$$

For by (4.1) we see that

$$
\lim _{n \rightarrow \infty}(-1)^{n} \frac{\Lambda_{n}\left(x_{0}\right) \pi^{2 n}}{\sin \pi x_{0}}=\frac{2}{\pi} \quad\left(0<x_{0}<1\right) .
$$

THEOREM 4.4. There exists a constant $M$ such that

$$
0 \leqq(-1)^{n} \int_{0}^{1} G_{n}(x, t) d t \leqq \frac{M}{\pi^{2 n}} \quad(0 \leqq x \leqq 1 ; n=1,2, \cdots) .
$$

This is proved from equation (2.1) as Theorem 4.1 was proved from equation (3.2).

5. Lidstone series. We denote the class of real entire functions of exponential type less than $\pi$ by $P$. In order to make our results independent of the theory of entire functions we may introduce the class by means of the following definition.

Definition 5.1. A real entire function $f(x)$ belongs to the class $P$ if there exists a positive number $p<\pi$ 'such that 


$$
f^{(n)}(0)=O\left(p^{n}\right)
$$$$
(n \rightarrow \infty) \text {. }
$$

Concerning this class of functions we prove a preliminary result.

Lemma 5.1. If $f(x)$ belongs to $P$ then there exists a positive number $p<\pi$ such that

uniformly in $0 \leqq x \leqq 1$.

$$
f^{(n)}(x)=O\left(p^{n}\right)
$$

For, by Taylor's expansion

By (5.1)

$$
f^{(n)}(x)=\sum_{k=0}^{\infty} f^{(n+k)}(0) \frac{x^{k}}{k !}
$$

$$
\left|f^{(n)}(x)\right| \leqq M \sum_{k=0}^{\infty} p^{n+k} \frac{x^{k}}{k !} \leqq M p^{n} e^{p} \quad(0 \leqq x \leqq 1) ，
$$

where $M$ is some constant. This proves the lemma. Note that if $f(x)$ belongs to $P$ then $f(x+a)$ does also for every constant $a$. The interval $(0,1)$ of the lemma could be replaced by any finite interval.

Theorem 5.1. If $f(x)$ belongs to $P$, then (5.2) $f(x)=f(1) \Lambda_{0}(x)+f(0) \Lambda_{0}(1-x)+f^{\prime \prime}(1) \Lambda_{1}(x)+f^{\prime \prime}(0) \Lambda_{1}(1-x)+\cdots$, the series converging uniformly in $0 \leqq x \leqq 1$.

Denote by $s_{n}(x)$ the sum of the first $n$ terms of the series (5.2). Then by Theorem 1.1, Theorem 4.4 and Lemma 5.2 there is a constant $M$ such that

Also

$$
\begin{aligned}
\left|f(x)-s_{2 n}(x)\right| \leqq \int_{0}^{1}(-1)^{n} G_{n}(x, t)\left|f^{(2 n)}(t)\right| d t \leqq M\left(\frac{p}{\pi}\right)^{2 n} & \\
& (n=0,1, \cdots) .
\end{aligned}
$$

$$
s_{2 n+1}(x)=s_{2 n}(x)+f^{(2 n)}(0) \Lambda_{n}(x) .
$$

By Theorem 4.2 and Definition 5.1 we see that

$$
\lim _{n \rightarrow \infty} f^{(2 n)}(0) \Lambda_{n}(x)=0
$$

uniformly in $0 \leqq x \leqq 1$, so that the theorem is established.

THEOREM 5.2. If the series

$$
b_{0} \Lambda_{0}(x)+a_{0} \Lambda_{0}(1-x)+b_{1} \Lambda_{1}(x)+a_{1} \Lambda_{1}(1-x)+\cdots
$$

converges for a single value of $x$ in $0<x<1$, it converges uniformly throughout that interval to a function $f(x)$. Then the series 


$$
b_{0}+a_{0}-\frac{b_{1}}{\pi^{2}}-\frac{a_{1}}{\pi^{2}}+\frac{b_{2}}{\pi^{4}}+\frac{a_{2}}{\pi^{4}}-\cdots
$$

converges and

$$
f^{(2 k)}(x)=b_{k} \Lambda_{0}(x)+a_{k} \Lambda_{0}(1-x)+b_{k+1} \Lambda_{1}(x)+a_{k+1}(1-x)+\cdots
$$

for $0 \leqq x \leqq 1$ and $k=0,1,2, \cdots$.

For, if the series (5.3) converges for $x=x_{0}$ then

$$
\lim _{n \rightarrow \infty} b_{n} \Lambda_{n}\left(x_{0}\right)=0, \quad \lim _{n \rightarrow \infty} a_{n} \Lambda_{n}\left(1-x_{0}\right)=0 .
$$

Then by Theorem 4.3

$$
b_{n}=O\left(\pi^{2 n}\right), \quad a_{n}=O\left(\pi^{2 n}\right) .
$$

This shows by (4.1) that the series

$$
\begin{aligned}
\sum_{n=0}^{\infty} b_{n}\left[\Lambda_{n}\left(x_{0}\right)-(-1)^{n}\right. & \left.\frac{2 \sin \pi x_{0}}{\pi^{2 n+1}}\right] \\
& +a_{n}\left[\Lambda_{n}\left(1-x_{0}\right)-(-1)^{n} \frac{2 \sin \pi x_{0}}{\pi^{2 n+1}}\right]
\end{aligned}
$$

converges absolutely. Subtracting from this the series (5.3) convergent for $x=x_{0}$ we see that the resulting series

$$
-\frac{2 \sin \pi x_{0}}{\pi} \sum_{n=0}^{\infty}(-1)^{n} \frac{b_{n}}{\pi^{2 n}}+(-1)^{n} \frac{a_{n}}{\pi^{2 n}}
$$

must converge. That is, (5.4) must converge. When $x_{0}$ is replaced by the variable $x$ in (5.6) and (5.7) it is clear that both series converge uniformly in $0 \leqq x \leqq 1$. The same must be true of their difference, the series (5.3).

Finally, to prove (5.5) we must prove the series (5.5) uniformly convergent in $0 \leqq x \leqq 1$. To see this we note that the series

$$
\begin{aligned}
\sum_{n=0}^{\infty} b_{n+k}\left[\Lambda_{n}(x)-(-1)^{n}\right. & \left.\frac{2 \sin \pi x}{\pi^{2 n+1}}\right] \\
& +a_{n+k}\left[\Lambda_{n}(1-x)-(-1)^{n} \frac{2 \sin \pi x}{\pi^{2 n+1}}\right],
\end{aligned}
$$

$$
2 \sin \pi x \sum_{n=0}^{\infty}(-1)^{n} \frac{b_{n+k}}{\pi^{2 n+1}}+(-1)^{n} \frac{a_{n+k}}{\pi^{2 n+1}}
$$

both converge uniformly in $0 \leqq x \leqq 1$ by Theorem 4.1 and by the convergence of (5.7). Hence the sum of the series (5.8) and (5.9), series (5.5), must also converge uniformly in $0 \leqq x \leqq 1$. 
6. Completely convex functions. Since the successive Lidstone polynomials alternate in sign on the interval $0 \leqq x \leqq 1$, it is natural to consider for expansion in Lidstone series functions whose even derivatives alternate in sign on that interval. We call such a function completely convex there.

Definition 6.1. A real function $f(x)$ is completely convex on the interval $a \leqq x \leqq b$ if it has derivatives of all orders there and if

$$
(-1)^{k} f^{(2 k)}(x) \geqq 0 \quad(a \leqq x \leqq b ; k=0,1,2, \cdots) .
$$

For example, the functions $\sin x$ and $\cos x$ are completely convex on the intervals $(0, \pi)$ and $(-\pi / 2, \pi / 2)$, respectively.

We now prove certain properties of functions of this class.

ThEOREM 6.1. If $f(x)$ is completely convex in $0 \leqq x \leqq 1$, then

$$
\begin{aligned}
& f^{(2 k)}(0)=O\left(\pi^{2 k}\right), \\
& f^{(2 k)}(1)=O\left(\pi^{2 k}\right)
\end{aligned}
$$

Consider the identity (3.1) for the present function $f(x)$. Since every term of the series is non-negative we have

$$
\begin{aligned}
& 0 \leqq f^{(2 k)}(0) \Lambda_{k}(x) \leqq f(x), \\
& 0 \leqq f^{(2 k)}(1) \Lambda_{k}(1-x) \leqq f(x) \quad(0 \leqq x \leqq 1 ; k=0,1, \cdots) .
\end{aligned}
$$

In particular, choose $x=1 / 2$ and apply Theorem 4.3 . This gives the relations (6.1) at once.

THEOREM 6.2. If $f(x)$ is completely convex in $0 \leqq x \leqq 1$, then there is a constant $M$ such that

$$
\begin{aligned}
& 0 \leqq(-1)^{k} f^{(2 k)}(x) \leqq M\left(\frac{\pi}{x}\right)^{2 k}, \\
& 0 \leqq(-1)^{k} f^{(2 k)}(x) \leqq M\left(\frac{\pi}{1-x}\right)^{2 k} \quad(k \rightarrow \infty) .
\end{aligned}
$$

For, if $f(x)$ is completely convex in $a \leqq x \leqq b$, then the same is true of

$$
F(x)=f(a+b x-a x)
$$

in $0 \leqq x \leqq 1$. By Theorem 6.1 we have for $0 \leqq a<b \leqq 1$

$$
\begin{aligned}
& F^{(2 k)}(0)=f^{(2 k)}(a)(b-a)^{2 k}=O\left(\pi^{2 k}\right), \\
& F^{(2 k)}(1)=f^{(2 k)}(b)(b-a)^{2 k}=O\left(\pi^{2 k}\right)
\end{aligned}
$$

Choosing first $a=0, b=x<1$ and then $a=x>0, b=1$ we obtain (6.3) from (6.4). One sees easily from (6.2) that $M$ is independent of $x$ in (6.3).

We introduce next a familiar result of $\mathrm{J}$. Hadamard $\left({ }^{6}\right)$ as

(6) See, for example, T. Carleman, Les Fonctions Quasi-Analytiques, Paris, 1912, p. 12. 
Lemma 6.1. If $f(x)$ is of class $C^{2}$ in $a \leqq x \leqq b$ and if

then

$$
\begin{gathered}
\max _{a \leqq x \leqq b}|f(x)|=M_{0}, \\
\max _{a \leqq x \leqq b}\left|f^{\prime \prime}(x)\right|=M_{2}
\end{gathered}
$$

$$
\left|f^{\prime}(x)\right| \leqq \frac{2 M_{0}}{b-a}+\frac{M_{2}(b-a)}{2} \quad(a \leqq x \leqq b) .
$$

By use of this result we can now prove

THEOREM 6.3. If $f(x)$ is completely convex in $a \leqq x \leqq b$ with $b-a>1$, then $f(x)$ belongs to class $P$ and equation (5.2) holds.

From (6.4) we have for a suitable constant $M$

$$
\left|f^{(2 k)}(x)\right| \leqq M\left(\frac{\pi}{b-x}\right)^{2 k} \quad(a \leqq x \leq b) .
$$

Choose a number $c$ so near $a$ that $b-c>1$. Then

$$
\left|f^{(2 k)}(x)\right| \leqq M\left(\frac{\pi}{b-c}\right)^{2 k} \quad(a \leqq x \leqq c) .
$$

Setting $\pi /(b-c)=p$ we have by Lemma 6.1

$$
\left|f^{(2 k+1)}(x)\right| \leqq \frac{2 M}{c-a} p^{2 k}+M \frac{(c-a)}{2} p^{2 k+2} .
$$

That is,

$$
f^{(k)}(x)=O\left(p^{k}\right) \quad(k \rightarrow \infty),
$$

uniformly in $(a \leqq x \leqq c)$. This shows that $f(x)$ is entire and that $f(x+a)$ belongs to $P$. It follows that $f(x)$ belongs to $P$ and the theorem is proved.

7. Minimal convex functions. The sufficient condition of Theorem 6.3 for the representation of a function in Lidstone series is not necessary. For example, the function $\sinh x$ is not completely convex in any interval; yet it has the Lidstone expansion

$$
\sinh x=\sinh 1 \sum_{n=0}^{\infty} \Lambda_{n}(x) .
$$

We shall see that a convergent Lidstone series with every term non-negative in $0 \leqq x \leqq 1$ defines a function which is completely convex there. But observe that $\sin \pi x$ has the same property. Yet it has no Lidstone representation since every term of the Lidstone series for this function is zero. To obtain conditions that are both necessary and sufficient we introduce a further definition. 
DEFINITION 7.1. A function $f(x)$ is a minimal completely convex function in the interval $0 \leqq x \leqq 1$ if it is completely convex there and if $f(x)-\epsilon \sin \pi x$ is not completely convex there for any positive $\epsilon$.

For example, the functions $f(x)=0$ and $f(x)=\sin x$ are minimal convex functions in $0 \leqq x \leqq 1$ but the function $f(x)=\sin \pi x$ is not.

THEOREM 7.1. If the series

$$
\begin{aligned}
& \sum_{n=0}^{\infty}(-1)^{n} b_{n} \Lambda_{n}(x)+(-1)^{n} a_{n} \Lambda_{n}(1-x), \\
& a_{n} \geqq 0, \quad b_{n} \geqq 0 \quad(n=0,1,2, \cdots)
\end{aligned}
$$

converges to $f(x)$, then $f(x)$ is a minimal completely convex function in the interval $0 \leqq x \leqq 1$.

Differentiating series (7.1) we obtain

$$
(-1)^{k \cdot k} f^{(2 k)}(x)=\sum_{n=0}^{\infty}(-1)^{n} b_{n+k} \Lambda_{n}(x)+(-1)^{n} a_{n+k} \Lambda_{n}(1-x) .
$$

Since the right-hand side is non-negative $f(x)$ is completely convex in $0 \leqq x \leqq 1$. By Theorem 4.2

$$
(-1)^{k} f^{(2 k)}(x) \leqq M \sum_{n=0}^{\infty}\left(a_{n+k}+b_{n+k}\right) \pi^{-2 n}=M \pi^{2 k} R_{k},
$$

where

$$
R_{k}=\sum_{n=k}^{\infty} \frac{a_{n}}{\pi^{2 n}}+\frac{b_{n}}{\pi^{2 n}} .
$$

By Theorem 5.2, $R_{k}$ is defined and tends to zero with $1 / k$. For a given positive number $\epsilon$ and a number $x_{0}$ between zero and one we can find an integer $k$ so large that

$$
M R_{k}-\epsilon \sin \pi x_{0}<0 .
$$

That is, the function

$$
(-1)^{k}[f(x)-\epsilon \sin \pi x]^{(2 k)}
$$

is negative at $x_{0}$. Hence $f(x)$ is a minimal completely convex function in $0 \leqq x \leqq 1$.

For our next result we need to prove a lemma.

LEMMA 7.1. If $f(x)$ and $-f^{\prime \prime}(x)$ are non-negative in $0 \leqq x \leqq 1$ and if $f\left(x_{0}\right)>\epsilon \pi$ for some number $x_{0}$ in that interval, then

$$
f(x)>\epsilon \sin \pi x \quad(01 \leqq x \leqq 1) .
$$


The result is obvious geometrically, but we give an analytical proof. If $0<x_{0} \leqq 1$ we have by the convexity of $f(x)$

$$
\begin{aligned}
& f(x) \geqq f(0)+[\epsilon \pi-f(0)] x_{0}^{-1} x . \quad\left(0 \leqq x \leqq x_{0}\right), \\
& f(x) \geqq\left[f(0)\left(x_{0}-x\right)+\epsilon \pi x\right] x_{0}^{-1} \quad\left(0 \leqq x \leqq x_{0}\right) \text {, } \\
& f(x)>\epsilon \pi x \geqq \epsilon \sin \pi x \quad\left(0 \leqq x \leqq x_{0}\right) \text {. }
\end{aligned}
$$

If $x_{0}=1$ the proof is complete. Otherwise by applying what has just been proved to the function $f(1-x)$ we see that (7.2) also holds in $x_{0} \leqq x \leqq 1$. Finally, if $x_{0}=0$ the above proof is applicable to the function $f(1-x)$.

THEOREM 7.2. If $f(x)$ is a minimal completely convex function in $0 \leqq x \leqq 1$, then it can be expanded in a convergent Lidstone series.

Define $s_{n}(x)$ as in $\S 5$. Then under the present hypothesis on $f(x)$ it is clear from equation (3.1) that

$$
s_{n}(x) \leqq f(x) \quad(0 \leqq x \leqq 1 ; n=0,1, \cdots),
$$

and that $s_{n}(x)$ is a non-decreasing function of $n$ for each $x$. Hence $s_{n}(x)$ tends to some function as $n$ becomes infinite. We wish to prove that its limit is $f(x)$. Suppose the contrary and assume that for some $x_{0}$ in $0 \leqq x \leqq 1$

$$
f\left(x_{0}\right)-\lim _{n \rightarrow \infty} s_{n}\left(x_{0}\right)=\Delta>0 .
$$

Then

$$
f\left(x_{0}\right)-s_{2 n}\left(x_{0}\right)=\int_{0}^{1} G_{n}\left(x_{0}, t\right) f^{(2 n)}(t) d t \geqq \Delta \quad(n=1,2, \cdots) .
$$

Since $f(x)$ is a minimal completely convex function, $f(x)-\epsilon \sin \pi x$ fails to be completely convex in $0 \leqq x \leqq 1$ for every positive $\epsilon$. Choose $\epsilon<\Delta /(\pi M)$, where $M$ is the constant of Theorem 4.4. Then there exists an integer $k$ and a number $x_{0}$ in $(0,1)$ such that

$$
(-1)^{k} f^{(2 k)}\left(x_{0}\right)-\epsilon \pi^{2 k} \sin \pi x_{0}<0 .
$$

By virtue of Lemma 7.1 this implies that

$$
(-1)^{k} f^{(2 k)}(x) \leqq \epsilon \pi^{2 k+1}
$$$$
(0 \leqq x \leqq 1),
$$

so that by Theorem 4.4

$$
\int_{0}^{1} G_{k}\left(x_{0}, t\right) f^{(2 k)}(t) d t \leqq \epsilon \pi M<\Delta .
$$

This contradicts inequality (7.3). The assumption that $s_{n}(x)$ does not approach $f(x)$ as $n$ becomes infinite is untenable, so that our theorem is proved.

8. Necessary and sufficient conditions for representation. We conclude by 
proving a necessary and sufficient condition that $f(x)$ can be represented by an absolutely convergent Lidstone series

THEOREM 8.1. A necessary and sufficient condition that $f(x)$ can be represented by an absolutely convergent Lidstone series is that it should be the difference of two minimal completely convex functions on $0 \leqq x \leqq 1$.

To prove the sufficiency of the condition let

$$
f(x)=g(x)-h(x),
$$

where $g(x)$ and $h(x)$ are both minimal completely convex functions on $0 \leqq x \leqq 1$. By Theorem 7.2

$$
\begin{aligned}
& g(x)=\sum_{n=0}^{\infty} g^{(2 n)}(1) \Lambda_{n}(x)+g^{(2 n)}(0) \Lambda_{n}(1-x), \\
& h(x)=\sum_{n=0}^{\infty} h^{(2 n)}(1) \Lambda_{n}(x)+h^{(2 n)}(0) \Lambda_{n}(1-x) .
\end{aligned}
$$

Each series has only positive terms so that when we subtract them the result is an absolutely convergent Lidstone series whose sum is $f(x)$.

Conversely, assume that

$$
f(x)=\sum_{n=0}^{\infty} b_{n} \Lambda_{n}(x)+a_{n} \Lambda_{n}(1-x),
$$

the series converging absolutely. Set

$$
\begin{aligned}
g(x)= & \sum_{n=0}^{\infty}(-1)^{n}\left|b_{n}\right| \Lambda_{n}(x)+(-1)^{n}\left|a_{n}\right| \Lambda_{n}(1-x), \\
h(x)= & \sum_{n=0}^{\infty}(-1)^{n}\left\{\left|b_{n}\right|-(-1)^{n} b_{n}\right\} \Lambda_{n}(x) \\
& +(-1)^{n}\left\{\left|a_{n}\right|-(-1)^{n} a_{n}\right\} \Lambda_{n}(1-x) .
\end{aligned}
$$

These series both converge since (8.1) converges absolutely. Every term of these two series is positive. Hence by Theorem $7.1 g(x)$ and $h(x)$ are minimal completely convex. Since $f(x)=g(x)-h(x)$, our result is completely proved.

HARVARD UNIVERSITY

Cambridge, Mass. 